\title{
ON THE FAILURE OF THE WEAK BETH PROPERTY
}

\author{
RICHARD GOSTANIAN AND KAREL HRBACEK
}

\begin{abstract}
A general method for showing the failure of the weak Beth definability property for certain pairs of logics is discussed. Applications are made to the game logic, various infinitary logics and partially ordered quantifier logics.
\end{abstract}

In [5], Craig essentially shows that the satisfaction relation for second order logic is implicitly but not explicitly definable in second order logic-thereby showing that the analogue of Beth's definability theorem fails for second order logic. (See also Kreisel's review [10].) In this note we shall elaborate on Craig's idea to obtain the failure of the Beth property in various other situations. In so doing we shall obtain some new results as well as give some new proofs of old results.

All the logics we deal with are assumed to be such that their formulas are coded by sets in some standard way. In particular, we assume that the variables are indexed by ordinals, that $v_{\alpha}$ is coded by $(0, \alpha)$ and that each logic contains a binary relation symbol $\bar{E}$ such that atomic formulas of the form $\bar{E}\left(v_{\alpha}, v_{\beta}\right)$ are coded by $(1, \alpha, \beta)$.

The results we obtain actually show that an even weaker property than the usual Beth property fails. Following Friedman [6], we say that a logic $L_{1}$ has the weak Beth property with respect to a logic $L_{2}$ (Weak Beth $\left(L_{1}, L_{2}\right)$ ) if for every sentence $\varphi$ of $L_{1}$, of type $\mu \cup\{\bar{R}\}$, such that each structure $\Re$ of type $\mu$ has exactly one expansion $\langle\mathscr{T}, R\rangle$ which is a model of $\varphi$, there is a formula $\psi$ of $L_{2}$, of type $\mu$, such that

$$
\vDash \varphi \rightarrow\left(\forall v_{1}\right) \cdots\left(\forall v_{n}\right)\left[\bar{R}\left(v_{1}, \ldots, v_{n}\right) \leftrightarrow \psi\left(v_{1}, \ldots, v_{n}\right)\right] .
$$

The usual Beth property, Beth $\left(L_{1}, L_{2}\right)$, is obtained from the above by weakening the assumption "exactly one" to "at most one".

Using the method of this paper, it is possible to prove a general theorem asserting the failure of the weak Beth property for a large class of pairs of logics. However to do so would involve some cumbersome exposition which we would rather avoid. Hence we shall restrict ourselves to a detailed proof of the most involved case and then indicate the changes necessary to prove our other results.

We shall assume that the reader is familiar with the infinitary logics $L_{\kappa \lambda}$. The logics $L_{\kappa \lambda}^{G}$ are obtained from $L_{\kappa \lambda}$ by adding the game quantifier. That is,

Received by the editors February 7, 1975 and, in revised form, October 23, 1975.

AMS (MOS) subject classifications (1970). Primary 02B25, 02B20; Secondary 02H10.

- American Mathematical Society 1976 
the formation rules of $L_{\kappa \lambda}^{G}$ are those of $L_{\kappa \lambda}$ with the addition of: if $\varphi_{n}$ are formulas of $L_{\kappa \lambda}^{G}$, and $x_{n}$ are variables then $\left(\forall x_{0}\right)\left(\exists x_{1}\right)\left(\forall x_{2}\right)\left(\exists x_{3}\right) \cdots$ $\mathbb{M}_{n \in \omega} \varphi_{n}\left(x_{0}, \ldots, x_{2 n}\right)$ is a formula of $L_{\kappa \lambda}^{G}$ provided it has less than $\lambda$ free variables and $x_{i}$, for $i>2 n$, does not occur free in $\varphi_{n}$. $\left(L_{\infty \omega}^{G}\right.$ is then what is called $L_{\infty G}$ in [2].) A detailed definition of satisfaction for such logics may be found e.g. in Keisler [9].

TheOREM 1. Weak Beth $\left(L_{\omega_{1} \omega}^{G}, L_{\infty \infty}^{G}\right)$ fails.

Proof. Let $\mu$ be the similarity type consisting of the single binary relation symbol $\bar{E}$, and let $\varphi_{1}$ be the conjunction of

(i) $\mathrm{KP}$, and

(ii) $\sim\left(\exists v_{0}\right)\left(\exists v_{1}\right) \cdots \mathbb{M}_{n \in \omega} \bar{E}\left(v_{n+1}, v_{n}\right)$,

where KP is the Kripke-Platek set of axioms for admissible sets (see Barwise [2]). Note that $\varphi_{1}$ is a sentence of $L_{\omega_{1} \omega}^{G}[\mu]$ and that each model of $\varphi_{1}$ is wellfounded (and hence isomorphic to a transitive admissible set).

Let $\langle A, E\rangle$ be a model of $\varphi_{1}$. Using induction over the well-founded relation $E$, it is easy to show that there is a unique binary relation $R_{A}$ on $A$ such that $(x, f) \in R_{A}$ iff

$(0)$ in $\langle A, E\rangle, x$ is a formula of $L_{\infty \infty}^{G}[\mu]$ and $f$ is a function whose domain is an ordinal

and one of the following inductive clauses holds:

(1) in $\langle A, E\rangle, x=(1, \alpha, \beta)$ and $\alpha, \beta \in \operatorname{dom}(f)$ and $f(\alpha) \in f(\beta)$,

(2) in $\langle A, E\rangle, x=\sim y$ and all indices of the free variables of $y$ belong to $\operatorname{dom}(f)$ and $(y, f) \notin R_{A}$,

(3) in $\langle A, E\rangle, x=\wedge Y$ and for all $y \in Y,(y, f) \in R_{A}$,

(4) in $\langle A, E\rangle, x=(\exists Z) y$ where $Z$ is a set of variables and there is a function $g$ such that $f$ and $g$ agree on the indices of the free variables of $x$, $\operatorname{dom}(g)$ is an ordinal containing the indices of all variables in $Z$ and $(y, g) \in R_{A}$,

(5) in $\langle A, E\rangle, x=\left(\forall z_{0}\right)\left(\exists z_{1}\right)\left(\forall z_{2}\right)\left(\exists z_{3}\right) \cdots \mathbb{A}_{n \in \omega} y_{n}$ and if $f^{\left(v_{0}, \ldots, v_{2 n}\right)}$ is the function defined (in $\langle A, E\rangle$ ) by

$$
f^{\left(v_{0}, \ldots, v_{2 n}\right)}(\alpha)=\left\{\begin{array}{l}
v_{\alpha} \text { if } \alpha \text { is the index of } z_{k} \text { and } 0 \leqslant k \leqslant 2 n, \\
f(\alpha) \text { otherwise, }
\end{array}\right.
$$

then (in the real world) for every $v_{0}$ there is $v_{1}$ such that for every $v_{2}$ there is $v_{3} \ldots$ such that for all $n \in \omega,\left(y_{n}, f^{\left(v_{0}, \ldots, v_{2 n}\right)}\right) \in R_{A}$.

Next observe that some simple modifications of the proof of Barwise's Lemma 2.4 in [1] show that all syntactical notions of $L_{\infty \infty}^{G}[\mu]$ (in the sense of $\langle A, E\rangle)$ are uniformly $\left(\Delta_{1}\right)$ definable over all models of $\varphi_{1}$. This definability enables us to express conditions (0)-(4) by formulas of $L_{\omega \omega}[\mu \cup\{\bar{R}\}]$ and condition (5) by a formula of $L_{\omega_{1} \omega}^{G}[\mu \cup\{\bar{R}\}]$. Hence, if $\varphi_{2}(\bar{R})$ is

$$
(\forall x)(\forall f)[\bar{R}(x, f) \equiv(0) \wedge((1) \vee(2) \vee(3) \vee(4) \vee(5))],
$$

then $\langle A, E, R\rangle \vDash \varphi_{1} \wedge \varphi_{2}(\bar{R})$ iff $R=R_{A}$.

Moreover, if $\langle A, \in, R\rangle$ is a transitive model of $\varphi_{1} \wedge \varphi_{2}(\bar{R})$ and $A$ has the property:

(P) if $a \in A, b \subseteq A$ and $\bar{b} \leqslant a \overline{\bar{a}}$, then $b \in A$, 
then $R$ will be the satisfaction relation for $L_{\infty \infty}^{G}[\mu] \cap A$ over $A$, i.e., for $\xi, f \in A,(\xi, f) \in R$ iff $\langle A, \in\rangle \vDash_{f} \xi$. The proof of this turns on the absoluteness of the syntactical notions (which are absolute because they are $\Delta_{1}$ ) as well as the fact that (P) insures that the $g$ in (4) will exist in $A$ if it exists at all.

Now let $\varphi(\bar{R})$ be $\left(\varphi_{1} \wedge \varphi_{2}(\bar{R})\right) \vee\left(\sim \varphi_{1} \wedge(\forall x)(\forall y) \bar{R}(x, y)\right)$. Then $\varphi(\bar{R})$ $\in L_{\omega_{1} \omega}^{G}[\mu \cup\{\bar{R}\}]$, and the above considerations immediately imply that for every structure $\langle A, E\rangle$ there is a unique $R$ such that $\langle A, E, R\rangle \vDash \varphi(\bar{R})$. Hence the hypothesis of the weak Beth property holds. To complete the proof it suffices to show that the conclusion is not satisfied by any formula $\psi$ of $L_{\kappa \kappa}^{G}[\mu]$ for any regular $\kappa$.

Hence assume that $\psi\left(v_{0}, v_{1}\right) \in L_{\kappa \kappa}^{G}[\mu]$ and is such that $\vDash \varphi(\bar{R}) \rightarrow\left(\forall v_{0}\right)$ $\left(\forall v_{1}\right)\left[\psi\left(v_{0}, v_{1}\right) \equiv \bar{R}\left(v_{0}, v_{1}\right)\right]$. Then if $H_{\kappa}$ is the set of all sets hereditarily of power less than $\kappa, H_{\kappa}$ is a transitive model of $\varphi_{1}$ and has property (P).

Hence

$$
\left\langle H_{\kappa}, \in, R\right\rangle \vDash \varphi(\bar{R})
$$

and so

$$
\left\langle H_{\kappa}, \in, R\right\rangle \vDash\left(\forall v_{0}\right)\left(\forall v_{1}\right)\left[\psi\left(v_{0}, v_{1}\right) \equiv \bar{R}\left(v_{0}, v_{1}\right)\right],
$$

where $R$ is the satisfaction relation for $L_{\kappa \kappa}^{G}[\mu]$ over $\left\langle H_{\kappa}, \in\right\rangle$.

The usual Tarski style diagonalization will now complete the proof. Indeed, let $\xi\left(v_{0}\right)$ be $\psi\left(v_{0},\left\{\left\langle 0, v_{0}\right\rangle\right\}\right)$, where it is understood that each occurrence of $\left\{\left\langle 0, v_{0}\right\rangle\right\}$ is replaced by a formula of $L_{\omega \omega}[\mu]$ using the standard definitions of \{\}$,\langle\rangle$, and 0 . Then if $a \in H_{\kappa}$ is $\sim \xi\left(v_{0}\right)$, it follows that

$$
\begin{gathered}
\left\langle H_{\kappa}, \in\right\rangle \vDash \xi(a) \operatorname{iff}\left\langle H_{\kappa}, \in\right\rangle \vDash \psi(a,\{\langle 0, a\rangle\}) \operatorname{iff}\left\langle H_{\kappa}, \in, R\right\rangle \vDash \bar{R}(a,\{\langle 0, a\rangle\}) \\
\operatorname{iff}(a,\{\langle 0, a\rangle\}) \in R \text { iff }\left\langle H_{\kappa}, \in\right\rangle \vDash \sim \xi(a) .
\end{gathered}
$$

This contradiction completes the proof. Q.E.D.

Next recall that in [13], Malitz has shown that Beth $\left(L_{\omega_{1} \omega_{1}}, L_{\infty \infty}\right)$ fails. His proof, as given, does not show the failure of Weak Beth $\left(L_{\omega_{1} \omega_{1}}, L_{\infty}\right)$, but can be easily modified to do so. If, however, we replace $L_{\infty \infty}^{G}$ by $L_{\infty \infty}$ everywhere in the proof of Theorem 1, ignore (5) and note that $\varphi_{1}$ is also a sentence of $L_{\omega_{1} \omega_{1}}$, we obtain a new proof of this result, i.e.,

Theorem 2. Weak Beth $\left(L_{\omega_{1} \omega_{1}}, L_{\infty \infty}\right)$ fails.

Continuing in the same vein, we recall that Gregory [8] has shown that Beth $\left(L_{\kappa^{+} \omega}, L_{\kappa^{+} \kappa}\right)$ fails for all regular $\kappa>\omega$ and actually (with the use of a later result of Tait) for all $\kappa$ such that cf $(\kappa)>\omega$. Gregory's proof however, unlike the above mentioned proof of Malitz, does not appear capable of modification to show the failure of the corresponding weak Beth property. Our method, however, does do this, and indeed provides an even stronger result. To show this we first need some definitions.

Definition. An ordinal $\alpha$ is called $L_{\kappa \lambda}$ accessible if there is a sentence $\varphi(\bar{U}, \bar{L}, \ldots)$ of $L_{\kappa \lambda}$ such that

(I) if $\langle A, U,\langle, \ldots\rangle \vDash \varphi$, then $<$ well-orders $U$, and

(II) there is an $\langle A, U,\langle, \ldots\rangle \vDash \varphi$ in which $\langle U,\langle\rangle$ has order type greater than or equal to $\alpha$. Such a sentence is sometimes said to pin down $\alpha$. 
Definition. If $A$ is a transitive set, $\operatorname{ord}(A)$ is the supremum of the ordinals in $A$.

\section{THEOREM 3.}

(a) Weak $\operatorname{Beth}\left(L_{\kappa^{+} \omega}, L_{\lambda \lambda}\right)$ fails for all regular $\lambda$ which are $L_{\kappa^{+} \omega}$ accessible.

(b) Weak $\operatorname{Beth}\left(L_{\kappa^{+} \omega}, L_{\infty \omega} \cap A\right)$ fails for all admissible $A$ such that ord $(A)$ is $L_{\kappa^{+} \omega}$ accessible.

Proof. (a) Replace (ii) in the proof of Theorem 1 by

(ii') $\varphi_{0}(U,<, \ldots) \wedge\left(\forall v_{0}\right)\left(\forall v_{1}\right)\left[\bar{E}\left(v_{0}, v_{1}\right) \rightarrow \bar{F}\left(v_{0}\right) \bar{F}\left(v_{1}\right)\right]$ where $\varphi_{0}$ is an $L_{\kappa^{+} \omega}$ sentence which pins down $\lambda, \bar{E}$ is the binary relation symbol of (i) and $\bar{F}$ is a unary function symbol. We let $\mu$ be the similarity type containing all the symbols of (i) and (ii').

If $\varphi_{1}$ is the conjunction of (i) and (ii'), then each structure $\langle A, E, U$, $\langle, F, \ldots\rangle$ which is a model of $\varphi_{1}$ is such that $\langle A, E\rangle$ is well-founded. Furthermore, $\left\langle H_{\lambda}, \in\right\rangle$ can be expanded to a model of $\varphi_{1}$. Since $\lambda$ is regular, $H_{\lambda}$ has the property (P), so that the rest of the proof now follows the pattern of the proof of Theorem 1-again ignoring clause (5).

Part (b) is proven in the same way as part (a) except that the property (P) is not necessary here. Q.E.D.

Our promised strengthening of Gregory's result now follows as an easy corollary of Theorem 3(a). We remark that it was also obtained by Friedman (unpublished) using a very complicated argument.

Corollary 3. Weak Beth $\left(L_{\kappa^{+} \omega}, L_{\kappa^{+} \kappa^{+}}\right)$fails for all $\kappa$ with $\mathrm{cf}(\kappa)>\omega$.

Proof. If $\mathrm{cf}(\kappa)>\omega$ then $\kappa^{+}$is $L_{\kappa^{+} \omega}$ accessible as is shown in [3] or [4]. Q.E.D.

In connection with Corollary 3 we should mention that Gregory [8] has also shown that Beth $\left(L_{\kappa^{+} \omega}, L_{\infty \kappa}\right)$ fails for all regular $\kappa>\omega$. The failure of the weak Beth property for this situation does not seem susceptible to our method and is still open. ${ }^{1}$

The proofs of Theorems 2 and 3 are simple modifications of the ideas in the proof of Theorem 1. We remark that another easy modification of these ideas would show that Weak Beth $\left(L_{\kappa \omega}, L_{2^{\kappa^{+}}}\right)$fails for all regular $\kappa$, if there were an $L_{\kappa \omega}$ sentence $\varphi_{0}$ such that (I) and (II') there is a structure $\langle A, U,<, \ldots\rangle$ which is a model of $\varphi_{0}$ in which $\langle U,\langle\rangle$ has order type greater than or equal to $\left(2^{\kappa}\right)^{+}$. However, since Beth $\left(L_{\kappa \omega}, L_{(2 \kappa)^{+\kappa}}\right)$ holds (see Malitz [13]) it follows that there can be no such sentence $\varphi_{0}$, so that we have another proof of the result of Lopez-Escobar [11]:

The class of all well-orderings is not $P C_{\Delta}$ in any logic $L_{\kappa \omega}$.

Finally, we shall apply our methods to the simplest of the partially ordered quantifier logics introduced by Henkin, i.e., the extension of $L_{\omega \omega}$ by the quantifier

$$
\left(\begin{array}{ll}
\forall x & \exists y \\
\forall z & \exists w
\end{array}\right)
$$

${ }^{1}$ However, S. Shelah announced that Weak Beth $\left(L_{\kappa^{+} \omega}, L_{\infty \lambda^{+}}\right)$fails when $\kappa=2^{\lambda^{+}}$(see Notices Amer. Math. Soc. 22 (1975). Abstract A-474). 
We shall denote this logic by $L^{H}$ and refer the reader to [12] for a detailed description. In [12] it is shown that $L^{H}$ fails to have the interpolation property. Here we prove

THEOREM 4. Weak Beth $\left(L^{H}, L^{H}\right)$ fails.

Proof. Ehrenfeucht has shown that there is a sentence $\varphi_{0}$ of $L^{H}$ all of whose models are isomorphic to $\langle\omega,\langle\rangle$ (see [12]). Using this, it is easy to write down a sentence $\varphi_{1}(\bar{E})$ of $L^{H}$, all of whose models are isomorphic to $\left\langle H_{\omega}, \in\right\rangle$. Now proceed to define inductively the relation $R_{A}$, with clauses (3) and (4) limited to finite conjunctions and quantifiers and clause (5) replaced by

$\left(5^{\prime}\right)$ in $\langle A, E\rangle$,

$$
x=\left(\begin{array}{ll}
\forall z_{0} & \exists z_{1} \\
\forall z_{2} & \exists z_{3}
\end{array}\right) y
$$

and if $f^{\left(v_{0}, v_{1}, v_{2}, v_{3}\right)}$ is the function defined by

$$
f^{\left(v_{0}, v_{1}, v_{2}, v_{3}\right)}(\alpha)=\left\{\begin{array}{l}
v_{k} \text { if } \alpha \text { is the index of } z_{k} \text { and } 0 \leqslant k \leqslant 4 \\
f(\alpha) \text { otherwise, }
\end{array}\right.
$$

then (in real world) for every $v_{0}$ there is $v_{1}$ and for every $v_{2}$ there is $v_{3}$ depending only on $v_{2}$ such that $\left(y, f^{\left(v_{0}, v_{1}, v_{2}, v_{3}\right)}\right) \in R_{A}$.

The rest of the proof is now essentially the same as the proof of Theorem 1 .

In closing, we would like to add that further applications of the method of this paper can be found in [7].

\section{REFERENCES}

1. J. Barwise, Infinitary logic and admissible sets, J. Symbolic Logic 34 (1969), 226-252.

2. _- Absolute logics and $L_{\infty}$, Ann. Math. Logic 4 (1972), 309-340. MR 49 \#2252.

3. J. Barwise and K. Kunen, Hanf numbers for fragments of $L_{\infty \omega}$, Israel J. Math. 10 (1971), 306-320. MR 47 \#4758.

4. C. C. Chang, Some remarks on the model theory of infinitary languages, Lecture Notes in Math., no. 72, Springer-Verlag, Berlin and New York, 1968, pp. 36-63.

5. W. Craig, Satisfaction for $n$-th order languages defined in $n$-th order languages, J. Symbolic Logic 30 (1965), 13 -25. MR 33 \#3883.

6. H. Friedman, Beth's theorem in cardinality logics, Israel J. Math. 14 (1973), 205-212. MR 47 \#6472.

7. R. Gostanian and K. Hrbacek, Propositional extensions of $L_{\omega_{1} \omega}$, Dissertationes Math. (submitted).

8. J. Gregory, Beth definability in infinitary languages, J. Symbolic Logic 39 (1974), 22-26.

9. H. J. Keisler, Formulas with linearly ordered quantifiers, Lecture Notes in Math., no. 72, Springer-Verlag, Berlin and New York, 1968, pp. 96-130.

10. G. Kreisel, Review of [5], Math. Rev. 33 (1967), 659-660.

11. E.G.K. Lopez-Escobar, On defining well-orderings, Fund. Math. 59 (1966), 13-21; ibid. 59 (1966), 299-300. MR 34 \#30; \#7354.

12. - A non-interpolation theorem, Bull. Acad. Polon. Sci. Sér. Sci. Math. Astronom. Phys. 17 (1969), 109-112. MR 39 \#3978.

13. J. Malitz, Infinitary analogs of theorems from first order model theory, J. Symbolic Logic 36 (1971), 216-228. 